\title{
Progression of abnormalities in adrenomyeloneuropathy and neurologically asymptomatic X-linked adrenoleukodystrophy despite treatment with "Lorenzo's oil"
}

B M van Geel, J Assies, E B Haverkort, J H T M Koelman, B Verbeeten Jr, R J A Wanders, P G Barth, on behalf of the Dutch X-ALD/AMN Study Group

Department of Neurology

B M van Geel

Departments of Internal Medicine and Psychiatry

J Assies

Department of Dietetics

E B Haverkort

Department of Neurology, Division of Clinical

Neurophysiology

J H T M Koelman

Department of Radiology

B Verbeeten, Jr

Department of Biochemistry

R J A Wanders

Departments of

Pediatrics and

Neurology, Academic

Medical Center,

University of

Amsterdam,

Amsterdam, the

Netherlands

P G Barth

Correspondence to: Dr B M van Geel, Academic Medical Center, University of Amsterdam, Department of Neurology, PO Box 22700, $1100 \mathrm{DE}$

Amsterdam, the Netherlands. Telephone 0031205663842 fax 003120 6971438; email b.m.vangeel@amc.uva.nl.

Received 29 September 1998 and in revised form

28 January 1999

Accepted 4 February 1999

\begin{abstract}
Objectives-X-linked adrenoleukodystrophy (X-ALD) is an inherited disorder of peroxisomal fatty acid oxidation, biochemically characterised by the accumulation of saturated very long chain fatty acids (VLCFAs), particularly hexacosanoic acid (C26:0). Dietary treatment with a 4:1 mixture of glyceroltrioleate and glyceroltrierucate ("Lorenzo's oil") normalises plasma VLCFA concentrations, but neither ameliorates nor arrests the rapid progression of neurological symptoms in the cerebral variants of X-ALD. The efficacy of "Lorenzo's oil" in the milder phenotypes of X-ALD was assessed, as this has been much less investigated. Methods- Twenty two patients who were treated with "Lorenzo's oil" for at least 12 months for a median period of 2.5 (range 1.0-6.0) years were studied. Two had asymptomatic ALD, four the "Addison only" variant, 13 adrenomyeloneuropathy (AMN), and three were symptomatic
\end{abstract} female carriers.

Results-The plasma C26:0 concentration normalised or near normalised in 19 patients $(86 \%)$, in the three others it decreased significantly. Nevertheless, disability as measured with the extended disability status scale score increased mildly ( 0.5 (95\% confidence interval (95\% CI) $0.25-1.0)$ ) in the 16 patients with neurological symptoms. Furthermore, one "Addison only" patient and one patient with AMN developed cerebral demyelination, and another "Addison only" patient developed AMN. Adrenocortical insufficiency evolved in one patient with $A M N$, and hypogonadism in one patient with asymptomatic ALD and two patients with AMN. Nerve conduction, evoked potential studies (SEP, BAEP, VEP), and abnormalities on cerebral MRI did not improve. On the other hand, side effects were often noted-namely, mild increases in liver enzymes (55\%), thrombocytopenia (55\%), gastrointestinal complaints (14\%), and gingivitis (14\%). We also found a mild decrease in haemoglobin concentration and leucocyte count.

Conclusions-The data suggest that treatment with "Lorenzo's oil" neither improved neurological or endocrine function nor arrested progression of the disease.
Furthermore, the oil often induced adverse effects. Therefore, it is advocated that "Lorenzo's oil" should not be prescribed routinely to patients with X-ALD who already have neurological deficits. (F Neurol Neurosurg Psychiatry 1999;67:290-299)

Keywords: X-linked adrenoleukodystrophy; adrenomyeloneuropathy; Lorenzo's oil; peroxisome

$\mathrm{X}$-Linked adrenoleukodystrophy (X-ALD) is an inherited disorder of peroxisomal metabolism, biochemically characterised by deficient $\beta$-oxidation of saturated very long chain fatty acids (VLCFAs), particularly hexacosanoic acid (C26:0). ${ }^{1-3}$ The consequent accumulation of these fatty acids is associated with demyelination within the CNS, and with adrenocortical insufficiency and hypogonadism. ${ }^{45}$

The phenotypic expression of X-ALD is highly variable, with adrenomyeloneuropathy (AMN) and childhood cerebral ALD (CCALD) accounting for $70 \%$ to $80 \%$ of all cases. $^{5-8}$ In AMN, the spinal cord and peripheral nerves are mainly affected, resulting in spastic paraparesis, sensory abnormalities in the legs, and bladder or anal sphincter dysfunction. Neurological symptoms emerge in the second to fourth decade, and the progression is relatively slow. Childhood cerebral ALD is characterised by progressive cerebral demyelination in previously healthy boys, resulting in a persistent vegetative state or death within 3 years after the onset of symptoms. In $10 \%$ to $15 \%$ of patients with X-ALD only adrenocortical insufficiency is demonstrable (the "Addison only" phenotype), and in $5 \%$ to $10 \%$ an excess of plasma VLCFAs is the only manifestation (asymptomatic ALD). At least 15 to $30 \%$ of female carriers develop AMN-like symptoms. ${ }^{79}$

As it was assumed that the excess of VLCFAs was toxic to myelin and the adrenal cortex and testis, several attempts were made to lower the plasma concentrations of VLCFAs. Restriction of VLCFA intake alone induced neither biochemical nor clinical improvement. ${ }^{10}$ The failure of this therapy seemed to be due to endogenous biosynthesis of VLCFAs via chain elongation of shorter chain fatty acids. ${ }^{11}$ Rizzo et al discovered that oleic acid (C18:1) reduced the concentrations of VLCFAs in cultured fibroblasts of patients 
with X-ALD, probably by competitive inhibition of the endogenous elongation system of saturated fatty acids. ${ }^{12}$ Oral administration of oleic acid in triglyceride form, glyceroltrioleate (GTO), significantly lowered the plasma concentration of VLCFAs. ${ }^{13}{ }^{14}$ In 1989 Rizzo et al reported that the combination of GTO and erucic acid (C22:1) in triglyceride form (glyceroltrierucate, or GTE) normalised the plasma concentration of C26:0 within 1 month in most patients with X-ALD. ${ }^{15}$ The combination of GTO and GTE in a 4:1 ratio became known as "Lorenzo's oil", a tribute to Lorenzo Odone, the first patient treated with the mixture. ${ }^{16}$

So far, several groups of investigators were unable to show any beneficial effect of "Lorenzo's oil" on the cerebral forms of X-ALD. ${ }^{17-20}$ Although the efficacy of the oil has been studied much less in patients with milder variants of X-ALD, many are still using "Lorenzo's oil". Aubourg et al reported in 1993 that the oil failed to induce a clinically relevant benefit in $\mathrm{AMN},{ }^{21}$ but this finding is yet to be confirmed in a comparable group of patients. As in other countries, a non-randomised trial was conducted in The Netherlands to study the efficacy of GTO/GTE therapy in X-ALD.

\section{Patients and methods}

STUDY DESIGN

Male patients with X-ALD and symptomatic heterozygotes known to members of the Dutch X-linked Adrenoleukodystrophy/ Adrenomyeloneuropathy Study Group were offered participation in an non-randomised trial with GTO/GTE dietary therapy between January 1990 and January 1995. They were scheduled to be seen every 3 months by a (paediatric) neurologist and endocrinologist, and a dietician. Blood samples were taken at 3 month intervals to study plasma VLCFAs, haematology, and the function of liver, kidneys, adrenal cortex, and testes. Furthermore, every 12 months electrophysiological studies (electroneurography and evoked potential studies) and cerebral MRI were performed. End points were discontinuation of treatment or death after at least 1 year of treatment, or the end of our observation period.

\section{PATIENTS}

Twenty two patients were followed up according to the study protocol, and their test results were used for calculations. Two patients were asymptomatic (ages 23.6 and 27.9 years), four had the "Addison only" phenotype (median age 5.5 years; range 2.9-31.3), 13 had AMN (median age 43.9 years; range 30.3-55.2), and three were symptomatic heterozygous women (median age 52.0 years; range 50.3-55.6). At the initiation of the GTO/GTE therapy, the median duration of neurological symptoms in the AMN group was 8.0 (range 0.8-33.0) years, and in the female carriers this was 13.5 (range 13.1-18.0) years. The median duration of adrenocortical insufficiency in the four patients with the "Addison only" phenotype was 0.7 (range $0.2-0.9$ ) years, and in the six of the 13 patients with AMN (46\%) and adrenocortical insufficiency this was 6.4 (range
3.3-11.8) years. The median follow up period of the 22 patients was 2.5 (range 1.0-6.0) years; the median duration of observation was 1.6 (range 1.2-1.9) years in the asymptomatic patients, 2.1 (range 1.0-2.7) years in the "Addison only" group, 3.5 (range 1.9-6.0) years in the patients with AMN, and 3.7 (range 1.3-4.0) years in the symptomatic heterozygous women.

Only limited data were available on another five patients, all with the "Addison only" phenotype. Their median age at the start of the dietary therapy was 12.5 (range 3.7-12.7) years, and the median duration of adrenocortical insufficiency 5.8 (range 3.4-8.5) years. The median period of follow up was 4.9 (range 3.0-6.9) years.

Fifteen others started treatment, but they were not included in the study for the following reasons: death and short observation period (two patients with $\mathrm{AMN}$ ); discontinuation of the diet due to rapid neurological deterioration (five patients with CCALD); and nonadherence to the diet or the follow up programme, or both (seven patients with AMN, one "Addison only" patient).

In all patients the diagnosis was confirmed by increased concentrations of C26:0 and C26:0/C22:0 ratios in plasma, ${ }^{22}$ cultured skin fibroblasts, ${ }^{23}$ or both. The study protocol was approved by the local medical ethics committees of the participating centres, and all participants or their parents gave their informed consent.

\section{DIETARY TREATMENT}

Aiming at the restriction of saturated VLCFA intake, only $10 \%-15 \%$ of the total caloric intake was provided as fat. Additionally, $4 \%$ of the energy intake consisted of GTE (Croda Universal, Ltd, Hull, UK), and $16 \%$ of GTO (Capital City Products Company, Columbus, $\mathrm{OH}, \mathrm{USA})$. To assess the adherence to the diet, patients or their parents were asked to keep a diary every 3 months for 7 days, and every 6 months they had to fill up a multiple choice questionnaire. This information was checked by the coordinating dietician $(\mathrm{EBH})$. From January 1992 onwards, plasma concentrations of oleic and erucic acid were also measured every 3 months to further corroborate compliance to the dietary treatment.

\section{CLINICAL STATUS}

Disability was assessed with the extended disability status scale (EDSS) ${ }^{24}$ and the Barthel index. $^{25}$ The EDSS score ranges from 0 (normal neurological examination) to 10 (dead), the Barthel index score ranges from 0 (totally dependent) to 20 (totally independent functioning). Handicap was rated according to the modified Rankin/Oxford handicap scale, ${ }^{26} \mathrm{a}$ score of 0 indicating no symptoms, and a score of 5 a severe handicap requiring constant attention. Detailed information about the scores is given in the table. 
LABORATORY INVESTIGATIONS

Plasma fatty acid concentrations

The VLCFA assays were performed as described earlier. ${ }^{2}$ Reference values in our laboratory are: $\mathrm{C} 26: 0,0.45-1.57 \mu \mathrm{mol} / 1 ; \mathrm{C} 24: 0 /$ C22:0 ratio, 0.57-0.92; C26:0/C22:0 ratio<0.01-0.02. Plasma concentrations of oleic acid (C18:1) ranged from 1035 to 2025 $\mu \mathrm{mol} / 1$ in healthy controls, and plasma concentrations of erucic acid (C22:1) ranged from 0 to $8 \mu \mathrm{mol} / 1$.

\section{Haematology and liver and renal function}

The reference values of our laboratory are as follows: haemoglobin $(\mathrm{Hb})$ concentration 8.0 $10.0 \mathrm{mmol} / 1$ for men and $7.5-9.8 \mathrm{mmol} / 1$ for women; leucocyte count $4.2-10.6 \times 10^{9} / 1$; platelet count $150-350 \times 10^{9} / 1$; plasma creatinine $75-110 \mu \mathrm{mol} / 1$ for men and $65-95 \mu \mathrm{mol} / 1$ for women; aspartate aminotransferase (ASAT, SGOT) $<47 \quad \mathrm{U} / 1$; alanine aminotransferase (ALAT, SGPT) $<37 \mathrm{U} / 1 ; \gamma$-glutamyl transferase $(\gamma-G T)<68 \mathrm{U} / 1$ for men and $<48 \mathrm{U} / 1$ for women; and alkaline phosphatase (AP) 26-103 $\mathrm{U} / 1$ for adults.

Endocrine function

The function of the adrenal cortex and testes was assessed as described previously. ${ }^{27}$ We used the following reference values: cortisol, $0.22-$ $0.65 \mu \mathrm{mol} / 1$ at 0900 ; dehydroepiandrosterone sulphate (DHEA-S) 2.0-10.0 $\mu \mathrm{mol} / \mathrm{l}$; adrenocorticotrophin (ACTH) 5-55 ng/l; testosterone 11.0-35.0 nmol/1; luteinising hormone (LH) 5-15 U/1; follicle stimulating hormone $(\mathrm{FSH})$ $1-10 \mathrm{U} / 1$

\section{ELECTRONEUROGRAPHY}

Motor and sensory conduction of the median, peroneal, and sural nerves, compound muscle action potential (CMAP), sensory nerve action potential (SNAP), median nerve and peroneal nerve $\mathrm{F}$ wave latencies, and soleus $\mathrm{H}$ reflex response were studied. The procedures and reference values of our laboratory were described recently. ${ }^{28}$ The test results of each investigation were additionally reviewed by an independent observer (JHTMK) and scored as (1) normal; (2) mildly abnormal; and, (3) markedly abnormal, based on the reference values of our laboratory.

\section{EVOKED POTENTIALS}

Somatosensory evoked potential (SEP)

Median nerve and posterior tibial nerve SEPs were elicited. Our procedures and reference values have been described previously. ${ }^{29}{ }^{30}$ The median nerve $\mathrm{N} 20$ and the posterior tibial

Assessment at baseline and at the end of the study

\begin{tabular}{|c|c|c|c|c|c|c|c|c|c|c|c|c|c|c|}
\hline \multirow[b]{2}{*}{ Patient } & \multirow[b]{2}{*}{ Phenotype } & \multirow[b]{2}{*}{$\begin{array}{l}\text { Age at } \\
t_{0}(y)\end{array}$} & \multirow[b]{2}{*}{$\begin{array}{l}\text { Duration } \\
\text { of therapy } \\
\text { (y) }\end{array}$} & \multicolumn{2}{|c|}{ Disability } & \multirow[b]{2}{*}{$\begin{array}{l}\text { Handicap } \\
\text { Rankin } \neq \\
t_{0} / t_{E}\end{array}$} & \multicolumn{2}{|c|}{ Endocrinologys } & \multicolumn{4}{|c|}{ Electrophysiology } & \multirow[b]{2}{*}{$\begin{array}{l}\text { Cerebral } \\
\text { MRIf } t_{0} / t_{E}\end{array}$} & \multirow[b]{2}{*}{ Remarks } \\
\hline & & & & $\begin{array}{l}E D S S^{\star} \\
t_{0} / t_{E}\end{array}$ & $\begin{array}{l}\text { Barthelf } \\
t_{0} / t_{E}\end{array}$ & & $\begin{array}{l}\text { Adrenal } \\
\text { cortex } \\
t_{0} / t_{E}\end{array}$ & $\begin{array}{l}\text { Testes } \\
t_{0} / t_{E}\end{array}$ & $\begin{array}{l}E N G \\
t_{0} / t_{E}\end{array}$ & $\begin{array}{l}S E P \\
t_{0} / t_{E}\end{array}$ & $\begin{array}{l}B A E P \\
t_{0} / t_{E}\end{array}$ & $\begin{array}{l}V E P \\
t_{0} / t_{E}\end{array}$ & & \\
\hline A & Asymp & 23.6 & 1.9 & $0.0 / 0.0$ & $20 / 20$ & $0 / 0$ & $\mathrm{n} / \mathrm{n}$ & $\mathrm{n} / \mathrm{n}$ & $1 / 1$ & $1 / 1$ & $2 / 2$ & $1 / 1$ & $1 / 1$ & Gingivitis \\
\hline B & Asymp & 27.9 & 1.2 & $1.5 / 0.0$ & $20 / 20$ & $0 / 0$ & $\mathrm{n} / \mathrm{n}$ & $\mathrm{n} / \mathrm{n}$ & $1 / 1$ & $1 / 1$ & $2 / 2$ & $1 / 1$ & $1 / 1$ & Stopped diet \\
\hline $\mathrm{C}$ & "Add-only" & 2.9 & 2.7 & $0.0 / 0.0$ & $20 / 20$ & $0 / 0$ & $\mathrm{i} / \mathrm{i}$ & $-1-$ & $1 / 1$ & $2 / 2$ & $2 / 1$ & $2 / 1$ & $1 / 3$ & $\begin{array}{l}\text { CNS demyelination, } \\
\text { BMT }\end{array}$ \\
\hline $\mathrm{D}$ & "Add-only" & 3.1 & 1.0 & $0.0 / 0.0$ & $20 / 20$ & $0 / 0$ & $\mathrm{i} / \mathrm{i}$ & $-1-$ & $1 / 1$ & $-1-$ & $-/-$ & $1 / 1$ & $1 / 1$ & BMT (elective) \\
\hline $\mathrm{E}$ & "Add-only" & 8.0 & 2.1 & $0.0 / 0.0$ & $20 / 20$ & $0 / 0$ & $\mathrm{i} / \mathrm{i}$ & $-1-$ & $1 / 1$ & $-/ 1$ & $1 / 1$ & $1 / 1$ & $1 / 1$ & \\
\hline $\mathrm{F}$ & "Add-only" & 31.3 & 2.1 & $1.0 / 1.5$ & $20 / 19$ & $0 / 1$ & $\mathrm{i} / \mathrm{i}$ & $\mathrm{n} / \mathrm{i}$ & $1 / 1$ & $1 / 2$ & $2 / 2$ & $1 / 1$ & $2 / 2$ & $\begin{array}{l}\text { AMN, hypogonadism, } \\
\text { GTE intolerance, } \\
\text { gingivitis }\end{array}$ \\
\hline G & $\mathrm{AMN}$ & 30.3 & 2.3 & $1.5 / 2.0$ & $20 / 20$ & $1 / 1$ & $\mathrm{n} / \mathrm{n}$ & $\mathrm{n} / \mathrm{i}$ & $2 / 2$ & $3 / 3$ & $3 / 3$ & $1 / 1$ & $1 / 1$ & $\begin{array}{l}\text { Hypogonadism, } \\
\text { gingivitis }\end{array}$ \\
\hline $\mathrm{H}$ & $\mathrm{AMN}$ & 31.9 & 2.6 & $5.0 / 6.0$ & $17 / 16$ & $3 / 2$ & $\mathrm{i} / \mathrm{i}$ & $\mathrm{i} / \mathrm{n}$ & $2 / 2$ & $3 / 3$ & $3 / 3$ & $1 / 1$ & $2 / 2+$ & \\
\hline I & $\mathrm{AMN}$ & 33.5 & 4.2 & $2.5 / 3.5$ & $20 / 20$ & $1 / 2$ & $\mathrm{i} / \mathrm{i}$ & $\mathrm{i} / \mathrm{i}$ & $3 / 3$ & $3 / \downarrow$ & $3 / \downarrow$ & $1 / 1$ & $1 / 3$ & CNS demyelination \\
\hline $\mathrm{J}$ & $\mathrm{AMN}$ & 34.7 & 5.1 & $2.5 / 2.5$ & $20 / 20$ & $2 / 2$ & $\mathrm{i} / \mathrm{i}$ & $\mathrm{n} / \mathrm{n}$ & $2 / 3$ & $3 / 3$ & $3 / 3$ & $1 / 1$ & $1 / 1$ & \\
\hline $\mathrm{K}$ & AMN & 35.5 & 6.0 & $5.5 / 6.5$ & $19 / 18$ & $2 / 3$ & $\mathrm{i} / \mathrm{i}$ & $\mathrm{i} / \mathrm{i}$ & $2 / 2$ & $3 / 3$ & $3 / 3$ & $1 / 1$ & $2 / 2$ & \\
\hline $\mathrm{L}$ & AMN & 39.0 & 2.2 & $3.5 / 2.0$ & $18 / 19$ & $2 / 2$ & $\mathrm{i} / \mathrm{i}$ & $-1-$ & $1 / 1$ & $3 / 3$ & $3 / 3$ & $1 / 1$ & $3 / 3$ & \\
\hline$M$ & $\mathrm{AMN}$ & 43.9 & 2.2 & $6.0 / 6.0$ & $20 / 18$ & $2 / 2$ & $\mathrm{n} / \mathrm{n}$ & $\mathrm{i} / \mathrm{i}$ & $3 / 3$ & $3 / 3$ & $3 / 3$ & $1 / 1$ & $2 / 2$ & \\
\hline $\mathrm{N}$ & $\mathrm{AMN}$ & 45.0 & 3.5 & $2.5 / 3.5$ & $20 / 20$ & $1 / 2$ & $\mathrm{n} / \mathrm{i}$ & $\mathrm{n} / \mathrm{n}$ & $2 / 2$ & $3 / 3$ & $3 / 3$ & $1 / 1$ & $1 / 1$ & $\begin{array}{l}\text { Adrenocortical } \\
\text { insufficiency }\end{array}$ \\
\hline $\mathrm{O}$ & $\mathrm{AMN}$ & 45.2 & 4.3 & $5.0 / 6.0$ & $-/ 18$ & $3 / 2$ & $\mathrm{i} / \mathrm{i}$ & $-/ \mathrm{i}$ & $2 / 2$ & $3 / 3$ & $1 / 3$ & $1 / 1$ & $-1-$ & \\
\hline $\mathrm{P}$ & $\mathrm{AMN}$ & 46.4 & 6.0 & $1.5 / 4.0$ & $19 / 19$ & $1 / 2$ & $\mathrm{i} / \mathrm{i}$ & $\mathrm{n} / \mathrm{n}$ & $2 / 3$ & $3 / 3$ & $3 / 3$ & $1 / 1$ & $1 / 1$ & \\
\hline Q & $\mathrm{AMN}$ & 46.7 & 1.9 & $6.5 / 7.0$ & $15 /-$ & $4 / 4$ & $\mathrm{n} / \mathrm{n}$ & $-1-$ & $1 / 1$ & $3 / 3$ & $3 / 3$ & $3 / \downarrow$ & $4 / 4$ & \\
\hline $\mathrm{R}$ & $\mathrm{AMN}$ & 52.7 & 1.9 & $5.5 / 5.0$ & $20 / 19$ & $3 / 3$ & $\mathrm{n} / \mathrm{n}$ & $-/ \mathrm{i}$ & $2 / 2$ & $3 / 3$ & $3 / 3$ & $1 / 1$ & $1 / 1$ & \\
\hline$S$ & $\mathrm{AMN}$ & 55.2 & 4.7 & $5.5 / 7.0$ & $19 / 14$ & $3 / 3$ & $\mathrm{n} / \mathrm{n}$ & $\mathrm{n} / \mathrm{i}$ & $3 / 3$ & $3 / 3$ & $3 / 3$ & $1 / 1$ & $2 / 2$ & Hypogonadism \\
\hline $\mathrm{T}$ & SHet & 50.3 & 4.0 & $8.0 / 8.0$ & $-/ 6$ & $4 / 4$ & $\mathrm{n} / \mathrm{n}$ & $-1-$ & $1 / 1$ & $1 / 1$ & $1 / 1$ & $1 / 1$ & $2 /-$ & \\
\hline $\mathrm{U}$ & SHet & 52.0 & 3.7 & $5.5 / 6.0$ & $20 / 18$ & $2 / 3$ & $\mathrm{n} / \mathrm{n}$ & $-1-$ & $2 / 2$ & -13 & $1 / 1$ & $-/ 1$ & $3 / 3$ & \\
\hline $\mathrm{V}$ & SHet & 55.6 & 1.3 & $5.5 / 6.0$ & $18 / 20$ & $2 / 2$ & $\mathrm{n} / \mathrm{n}$ & $-1-$ & $1 / 1$ & $3 / 3$ & $1 / 1$ & $1 / 1$ & $1 / 1$ & GTE intolerance \\
\hline
\end{tabular}

${ }^{\star}$ Extended disability status scale (EDSS) ${ }^{24}$ scores: $0=$ normal neurological examination; $1.0=$ no disability, minimal signs in one functional system $(\mathrm{FS}) ; 1.5=$ no disability, minimal signs in two FS; $2.0=$ minimal disability in one FS; $2.5=$ minimal disability in two FS; $3.0=$ no more than mild disability, fully ambulatory; $3.5=$ moderate disability though fully ambulatory; $4.0=$ self-sufficient for up to 12 hours despite severe disability; $4.5=$ requires minimal assistance, some limitations on activity, but able to work a full day; $5.0=$ severe disability, unable to perform full daily activities, but ambulatory without aid; $5.5=$ severe disability precluding full daily activities, able to walk $100 \mathrm{~m} ; 6.0=$ constant assistance required; $6.5=$ two canes or crutches required to walk; $7.0=$ unable to walk more than 5 m, essentially restricted to wheelchair; $7.5=$ restricted to wheelchair; $8.0=$ restricted to bed or chair.

+Barthel inde ${ }^{25}$ scores are the aggregate of bowel function (score, 0 to 2$)$, bladder function (0 to 2$)$, grooming $(0$ to 1$)$, toilet use $(0$ to 2$)$, feeding (0 to 2$)$, transfer ( 0 to 3 ), mobility ( 0 to 3 ), dressing ( 0 to 2 ), stairs walking ( 0 to 2 ), and bathing ( 0 to 1 ); the maximum score is 20 , indicating independent functioning.

$\ddagger$ Rankin/Oxford handicap scale ${ }^{26}: 0=$ no symptoms; $1=$ minor symptoms that do not interfere with lifestyle; $2=$ minor handicap; $3=$ moderate handicap; $4=$ moderately severe handicap; $5=$ severe handicap, totally dependent requiring constant attention.

Endocrine function: $\mathrm{n}=$ normal; $\mathrm{i}=$ insufficient; $-=$ not tested.

TElectrophysiological studies: $1=$ normal; $2=$ mildly abnormal; $3=$ clearly abnormal; $-=$ not tested; $\downarrow=$ worsening of markedly abnormal test results

$f$ Cerebral MRI: $1=$ normal; $2=$ mild focal abnormalities in basal ganglia, internal capsule or mesencephalic or pontine cortico spinal tracts; $3=$ moderate focal white matter abnormalities in basal ganglia, internal capsule, corticospinal tracts in mesencephalon and pons, or periventricular white matter; $4=$ extensive periventricular white matter abnormalities; $-=$ not performed; and, $+=$ lesions more pronounced without signs of progressive demyelination.

$\mathrm{ENG}=$ electroneurography; $\mathrm{SEP}=$ somatosensory evoked potential; $\mathrm{BAEP}=$ brainstem auditory evoked potential; $\mathrm{VEP}=\mathrm{visual}$ evoked potential; $\mathrm{Asymp}=\mathrm{asymptomatic}$ ALD; "Add-only"="Addison only" phenotype; $\mathrm{AMN}=$ adrenomyeloneuropathy; SHet=symptomatic heterozygote; $\mathrm{BMT}=$ bone marrow transplantation; $\mathrm{t}_{0}=\mathrm{baseline}$ assessment; $\mathrm{t}_{\mathrm{E}}=$ assessment at the end of follow up. 
nerve P37 latencies were particularly studied. The mean median nerve N20 latency (SD) was 19.5 (1.7) $\mathrm{ms}$, the mean posterior tibial nerve P37 latency (SD) was 40 (2.5) ms.

Brainstem auditory evoked potential (BAEP) Alternating clicks with an intensity of $75 \mathrm{~dB}$ above the hearing threshold with a maximum of $95 \mathrm{~dB}$ were delivered. The frequency of stimulation was $11 \mathrm{~Hz}$. Stimuli were presented monaurally with contralateral masking using a noise $40 \mathrm{~dB}$ above threshold. Two series of 2000 potentials were recorded, averaged, and superimposed with a Nicolet Pathfinder I (Nicolet Instruments Corporation, Madison, WI, USA). BAEPs were recorded with surface electrodes over the mastoids and referenced to $\mathrm{C}_{2}$. Electrode impedances were less than $5 \mathrm{k} \Omega$, the bandpass was $0.16-3.2 \mathrm{kHz}$, and the time of analysis was $10 \mathrm{~ms}$. We measured the I-V intervals. The upper limit of normal $\mathrm{I}-\mathrm{V}$ latency in adults in our laboratory is $4.43 \mathrm{~ms}$.

Visual evoked potential (VEP)

A full field pattern reversal paradigm was used. A high contrast chequerboard (square size $3.2 \times 3.2 \mathrm{~cm}$ ) was presented monocularly at a distance of $1.45 \mathrm{~m}$ with a stimulation rate of $1.3 \mathrm{~Hz}$. Two series of 100 stimuli presented to each eye were recorded, superimposed with a Nicolet Pathfinder I unit, and checked for reproducibility. Recording electrodes were placed over the midocciput and $5 \mathrm{~cm}$ laterally on both sides, and referenced to $\mathrm{C}_{\mathrm{z}}$. Electrode impedances were less than $5 \mathrm{k} \Omega$. The bandpass was $0.16-80 \mathrm{~Hz}$, and the time of analysis was $250 \mathrm{~ms}$. The P100 latencies were determined. The upper limit of normal P100 latency for adults in our laboratory is $118 \mathrm{~ms}$.

The BAEP, VEP, and SEP studies were reviewed by an independent observer (JHTMK) and scored as: (1) normal; (2) mildly abnormal; and (3) markedly abnormal, based on our reference values.

\section{NEURORADIOLOGY}

Most of the cerebral MRI was performed with a Siemens 1.5T unit (Siemens AG, Erlangen, Germany). Axial T2 weighted, proton density, and T1 weighted images without and with gadolinium enhancement were performed, and from January 1994 onwards sagittal T2 weighted imaging was carried out as well. The MR images were reviewed by two observers (BV and $\mathrm{BMvG}$ ) and scored as: (1) normal; (2) mild focal white matter abnormalities in basal ganglia, internal capsule, or corticospinal tracts in mesencephalon and pons; (3) moderate focal white matter abnormalities in basal ganglia, internal capsule, or mesencephalic and pontine corticospinal tracts; and (4) extensive periventricular white matter abnormalities.

\section{STATISTICAL ANALYSIS}

To detect significant changes in EDSS and Barthel and Rankin scores, the results were paired and the difference of the median and the $95 \%$ confidence interval ( $95 \% \mathrm{CI}$ ) were calculated using a Wilcoxon based non-parametric test. For the other variables the values at base- line were paired with those at 1 year of treatment and those at the end of treatment; to determine the significance of changes the mean of the differences and the $95 \%$ CI were calculated (Confidence Interval Analysis, British Medical Journal, London, UK).

\section{Results}

DIETARY TREATMENT

The table shows demographic data and results. According to the diaries, questionnaires, and the amount of dietary products used, all patients adhered strictly to the diet and GTO/ GTE supplementation. We found raised plasma concentrations of oleic and erucic acid in all but one patient ( $\mathrm{T}$ ), further corroborating the adherence to the dietary therapy. Two of the 22 patients developed severe gastrointestinal complaints ( $\mathrm{E}$ and $\mathrm{V}$ ), two others acute necrotising ulcerative gingivitis (F, G), and one patient (A) had both. This led to early discontinuation of dietary therapy in two (A and V).

\section{CLINICAL STATUS}

Disability as measured by an increase in the EDSS score progressed in 11 patients $(50 \%)$, and improved marginally in two of the 22 patients (9\%). The mean EDSS score of the 16 neurologically symptomatic patients at baseline was 4.5 and this increased mildly but significantly $(0.5 ; 95 \%$ CI $0.25-1)$. We did not see significant changes in the subscales of the EDSS (in particular the pyramidal, sensory, and bowel and bladder functions), Barthel index, or Rankin/Oxford handicap scale.

\section{LABORATORY INVESTIGATIONS}

Plasma VLCFA concentrations

We found a significant decrease in the plasma C26:0 concentration in all 22 patients, and normalisation or near normalisation in 19 $(86 \%)$. The mean plasma C26:0 concentration before the start of treatment was $2.76 \mu \mathrm{mol} / 1$ and decreased $1.02 \mu \mathrm{mol} / 1$ (95\% CI $0.54-1.50)$ after 3 months of treatment, $1.28 \mu \mathrm{mol} / 1$ (95\% CI $0.81-1.75)$ after 1 year, and $0.86 \mu \mathrm{mol} / 1$ (95\% CI 0.36-1.36) at the end of the observation period (fig 1). In three patients $(\mathrm{G}, \mathrm{H}$, and $S$ ), the plasma C26:0 concentrations in general were above $2.00 \mu \mathrm{mol} / 1$. The mean C24:0/ C22:0 ratio was 1.41 before "Lorenzo's oil" was started, and remained raised in all patients; the mean C24:0/C22:0 ratio decreased significantly to $0.40(95 \%$ CI $0.26-0.53)$ at 1 year of treatment, and to 0.34 (95\% CI $0.23-0.45)$ at the end of follow up. We found no changes in the mean C26:0/C22:0 ratio, which was 0.06 at baseline.

\section{Haematology}

One patient ( $\mathrm{R}$ ) developed unexplained anaemia; his $\mathrm{Hb}$ concentration recovered spontaneously. We found a significant decrease in mean $\mathrm{Hb}$ concentration, which was $9.3 \mathrm{mmol} / \mathrm{l}$ at baseline: $0.45 \mathrm{mmol} / 1$ (95\% CI $0.15-0.75)$ $\mathrm{mmol} / \mathrm{l}$ after 1 year of treatment, and 0.47 (95\% CI $0.16-0.78) \mathrm{mmol} / \mathrm{l}$ at the end of the observation period. The mean leucocyte count before the start of the dietary treatment was 


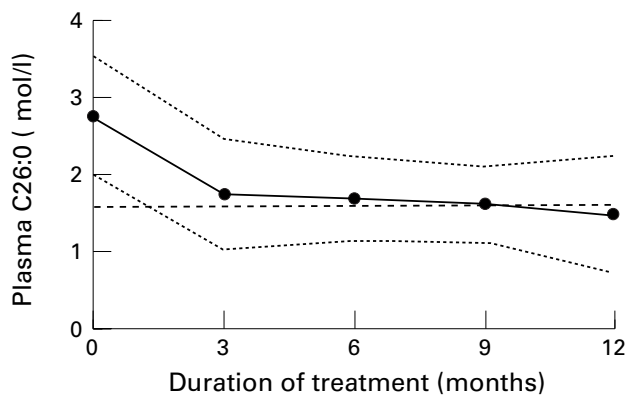

Figure 1 Mean plasma hexacosanoic acid (C26:0) concentration (solid line) $\pm S D$ (dotted lines) in the first year of treatment with "Lorenzo's oil". The horizontal dashed line indicates the upper limit of normal plasma C26:0 concentration $(1.57 \mu \mathrm{mol} / \mathrm{l})$. In all but three of the 22 patients the plasma C26:0 concentration became normal

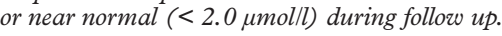

$7.1 \times 10^{9} / 1$, and at the end of follow up it was $1.2 \times 10^{9} / 1$ lower $(95 \%$ CI $0.1-2.2)$. In two patients (I and $\mathrm{M}$ ) we regularly found counts between 3.0 and $3.5 \times 10^{9} / 1$.

In 12 patients $(55 \%)$ the number of platelets fell to abnormally low concentrations, and in 10 of these patients the count was regularly between 50 and $100 \times 10^{9} / 1$. The lowest count was $51 \times 10^{9} / 1$ (patient I). None of the patients developed haemorrhagic diathesis. The mean platelet count before the use of the GTO/GTE dietary therapy was $232 \times 10^{9} / 1$, and decreased, with $63 \times 10^{9} / 1(95 \%$ CI $29-96)$ at 1 year of treatment, and with $76 \times 10^{9} / 1$ (95\% CI 46-105) at the end of follow up.

Liver and renal function

Mild increases in liver enzymes were seen during treatment in 12 of the 22 patients $(55 \%)$. Nine had a mild transient increase in plasma ALAT concentration ( $F, G, I-K, M, O, P$, and $\mathrm{R})$, and one (N) a mild transient increase in both plasma ASAT and ALAT. Two patients had a mild transient increase in plasma $\gamma$-GT concentration ( $\mathrm{J}$ and $\mathrm{U}$ ). The plasma AP concentration became mildly increased in four patients ( $B, G, M$, and $V)$. The mean plasma concentrations of ALAT, ASAT, $\gamma-\mathrm{GT}$, and AP did not change significantly. The plasma creatinine concentration remained normal in all patients, and the mean concentration did not change.

Endocrine function

One patient with AMN (N) developed adrenocortical insufficiency. In one of the "Addison only" patients $(\mathrm{F})$ and in four of the six patients with steroid hormone substitution therapy $(\mathrm{H}$, $\mathrm{I}, \mathrm{K}$, and $\mathrm{P}$ ) we saw greatly increased concentrations of plasma ACTH, regularly exceeding $2000 \mathrm{ng} / \mathrm{l}$ in two ( $\mathrm{H}$ and I) despite adequate substitution. There were no significant changes in the mean ACTH and cortisol concentrations. Plasma DHEA-S concentrations were not determined routinely in the adult male patients, but we measured extremely low values in 13 of $14(93 \%)$ men tested.

In a subset of 12 male patients in whom gonadal function was evaluated before the start of the diet, two had abnormally increased

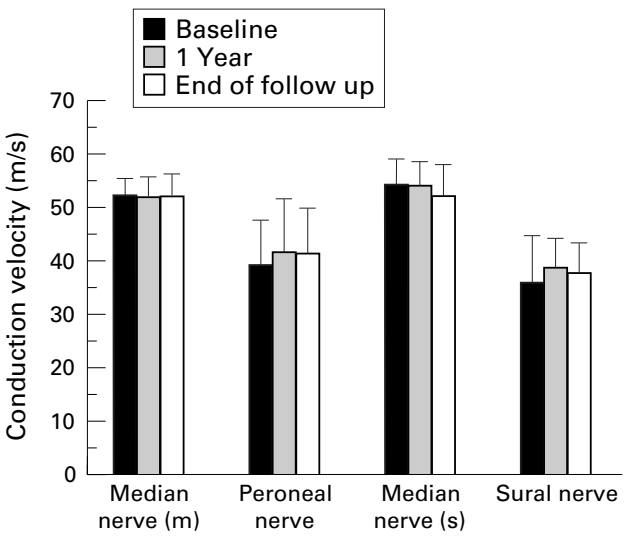

Figure 2 Mean values (SD) of motor nerve (median nerve $(m)$, peroneal nerve), and sensory nerve (median nerve (s), sural nerve) conduction at baseline, after 1 year of treatment, and at the end of the observation period in 13 patients with AMN and three symptomatic female carriers. patients with AMN and three symptomatic female carre velocities did not improve.

plasma concentrations of $\mathrm{LH}$ and FSH $(\mathrm{H}, \mathrm{M})$. Gonadal function improved in one of these patients $(\mathrm{H})$. During dietary treatment another three $(25 \%)$ developed gonadal insufficiency: in one patient $(F)$ the LH concentration became abnormally increased, in another (S) the FSH concentration increased above the upper limit of normal whereas the testosterone became abnormally low, and in one $(G)$ the plasma testosterone concentration became abnormally low. The mean plasma LH concentration ( $9.0 \mathrm{U} / 1$ at baseline) rose marginally but significantly (3.1 U/1 (95\% CI $0.3-6.0)$ ) at 1 year of dietary treatment; the increase at the end of the follow up period was not significant. The FA index was within the normal range in all patients at baseline, at 1 year of treatment, and at the end of the observation period, but the mean value had decreased significantly at the end of follow up (6.3 (95\% CI 0.7-11.9)). The mean concentrations of FSH, testosterone, and SHBG did not change.

\section{ELECTRONEUROGRAPHY}

In the six neurologically asymptomatic patients nerve conduction was normal at baseline and remained normal.

In the 16 neurologically symptomatic patients we found normal nerve conduction in four ( $\mathrm{L}, \mathrm{Q}, \mathrm{T}$, and $\mathrm{V}$ ), mild abnormalities in nine (G, H, J, K, N-P, R, and U), and marked abnormalities in three (I, M, and S). Most often, the results were compatible with a predominantly axonal sensorimotor polyneuropathy. ${ }^{28}$ During the follow up period the individual test results worsened in two patients $(\mathrm{J}$ and $\mathrm{P})$, but the motor and sensory nerve conduction velocities (fig 2) and other variables that were studied did not change significantly.

\section{EVOKED POTENTIALS}

SEP

The median nerve SEP remained normal in three of four neurologically asymptomatic patients tested ( $A, B$, and $F$ ); it normalised in the fourth patient (C). In six of the 13 AMN 

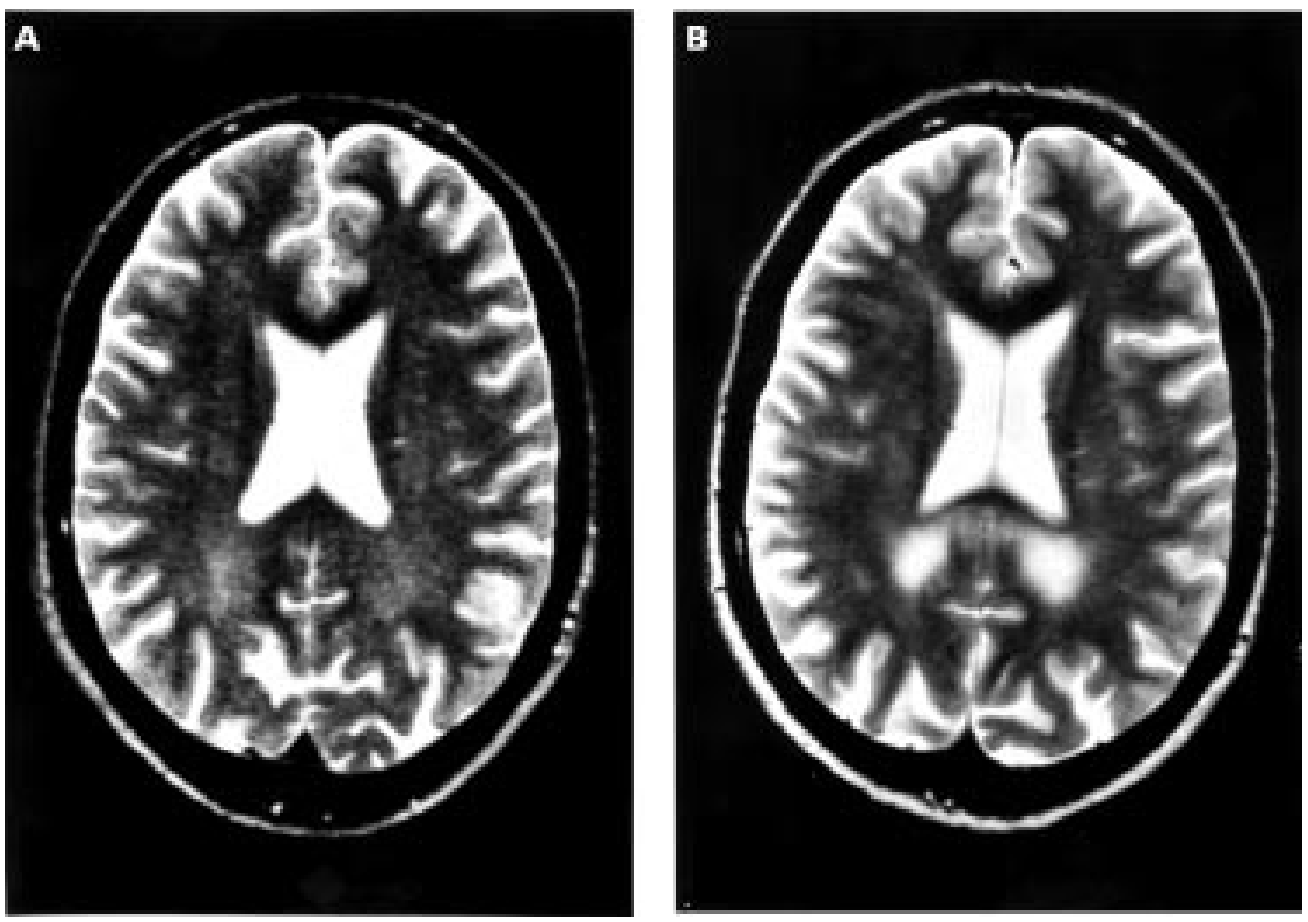

Figure 3 Development of occipital periventricular white matter abnormalities in patient I with AMN who used GTO and GTE compliantly for more than 4 years. The MRI of the brain before the start of treatment was unrevealing. At the left $(A)$, the cerebral T2 weighted MRI show mild hyperintensity after 1 year of treatment. At the right (B), the occipital demyelination is clearly visible 3 years later.

patients and the three heterozygotes the median nerve SEP was normal at baseline; it remained normal in five men (H-J, N, and Q) and the three female carriers (T-V), and became mildly abnormal in one patient with AMN (G). In seven patients with AMN the median nerve SEP was mildly delayed before the start of dietary treatment; it remained mildly abnormal in six (K, M, O, P, R, and S), and normalised in one (L). The mean value of the N20 latency (20 ms at baseline) did not change significantly.

The posterior tibial nerve SEP remained normal in two of three neurologically asymptomatic patients tested at baseline; one "Addison only" patient (F) developed a mildly abnormal posterior tibial nerve SEP. All but one (T) of the 16 symptomatic patients had markedly abnormal test results at baseline: in eight $(\mathrm{G}$, $\mathrm{H}, \mathrm{J}, \mathrm{N}, \mathrm{P}, \mathrm{R}, \mathrm{S}$, and $\mathrm{U}$ ) the $\mathrm{P} 37$ wave was absent. During follow up P37 responses eventually could be elicited in five of these patients (G, N, P, R, and S), but in two others (I and $M$ ) with a previously recognisable potential the SEP could not be detected any more. The mean of the P37 latency before the start of the dietary treatment $(52 \mathrm{~ms})$ did not change significantly.

\section{$B A E P$}

In the neurologically asymptomatic patients the BAEP remained normal in one patient $(E)$, mildly abnormal in three (A, B, and $\mathrm{J})$, and it normalised in one patient $(\mathrm{C})$. The 13 patients with $A M N$ had prolonged I-V intervals at baseline: in 12 the latencies remained markedly prolonged, and in one patient $(\mathrm{O})$ the $\mathrm{I}-\mathrm{V}$ interval changed from mildly to markedly abnormal. In the three female carriers (T-V) the intervals remained normal. We found no significant changes in the mean I-V latency during follow up.

\section{VEP}

The abnormal P100 latencies found at baseline in a boy with the "Addison only" phenotype (C) improved at the end of the observation period; in the patient with $A M N$ and severe occipital demyelination (Q) the prolonged P100 latency became even more markedly abnormal. There were no significant changes in mean P100 latency.

NEURORADIOLOGY

The MR images in the two asymptomatic patients were normal at baseline and at the end of the observation period. The oldest of the four "Addison only" patients (F) manifested mild focal abnormalities of the pontine corticospinal tracts, which did increase; another "Addison only" patient (C) developed demyelinating lesions in the splenium of the corpus callosum, and underwent bone marrow transplantation. ${ }^{31}{ }^{32}$ In the other two patients with this variant of X-ALD we found no signs of demyelination.

At baseline, cerebral MRI was performed in 12 patients with $\mathrm{AMN}$, and was abnormal in six $(50 \%)$ : four $(\mathrm{H}, \mathrm{K}, \mathrm{M}$, and $\mathrm{S})$ had mild abnormalities in the internal capsule, mesencephalon, pons, the basal ganglia or the periventricular white matter, one patient (L) had moderate focal white matter abnormalities, and one patient $(\mathrm{Q})$ manifested extensive lesions in the periventricular occipital white matter. During follow up, one patient (I) developed progressive demyelination in the occipital periventricular white matter (fig 3), and in 
another $(\mathrm{H})$ the lesions in the corticospinal tracts became more pronounced. We found no other significant changes. In a patient with $A M N$ with a pacemaker $(\mathrm{O})$ cerebral $C T$ was carried out, and showed no abnormalities at baseline and during follow up. One of the heterozygotes (U) showed moderate periventricular white matter abnormalities, and in another (V) we found no white matter lesions, both at baseline and at the end of follow up. In a claustrophobic female carrier (T) MRI was only performed before the start of treatment, and showed mild abnormalities in the mesencephalon.

OTHER PATIENTS

Limited data were available on five patients with the "Addison only" phenotype. In four the cerebral MRI remained normal; one patient developed abnormalities in the mesencephalic corticospinal tracts at the age of 11 years, and after more than 6 years of dietary therapy. Two patients developed thrombocyte counts below $10 \times 10^{9} / 1$ without concomitant haemorrhagic diathesis.

\section{Discussion}

Treatment with the GTO/GTE ("Lorenzo's oil") dietary therapy induced normal or near normal plasma concentrations of C26:0 in 19 of the 22 patients $(86 \%)$ we studied. Despite this significant biochemical response we were unable to detect a clinically relevant beneficial effect during a median follow up period of 2.5 years. Disability increased mildly but significantly in the 16 patients with neurological symptoms. One "Addison only" patient developed AMN (F), and another demyelinating lesions in the corpus callosum (C). One patient with AMN developed occipital demyelination (I), another (N) adrenocortical insufficiency, and three others $(\mathrm{F}, \mathrm{G}$, and $\mathrm{S}$ ) hypogonadism. Furthermore, we did not find improvement in the electrophysiological studies, endocrine function, and cerebral MR imaging.

Non-randomised studies in the United States, ${ }^{15}$ Italy, ${ }^{17} 18$ Japan, ${ }^{19}$ and Germany ${ }^{20}$ showed that the treatment with "Lorenso's oil" did not alter the downhill course in the phenotypes with cerebral demyelination despite the effective lowering of plasma VLCFA concentrations.

However, much less is known about the efficacy of the GTO/GTE diet in adult neurologically symptomatic patients. Cappa et al reported stability of neurological abnormalities in 11 patients with $A M N$ studied for a mean period of 2.0 years, and improvement of bladder function in two of these patients. ${ }^{18}$ Asano et al noted amelioration of spasticity in one patient with $\mathrm{AMN}$ followed up for 3 years. ${ }^{19} \mathrm{On}$ the other hand, Aubourg et al found clinical deterioration in nine of $14 \mathrm{AMN}$ patients over a 2.5 year period, although there was some improvement in peroneal nerve conduction and in posterior tibial nerve SEP; the clinical status of five symptomatic heterosygotes did not change. ${ }^{21}$ Korenke et al studied three AMN patients for 1.5 years: two men with AMN showed progression of neurological symptoms, and the third $A M N$ patient deteriorated mentally. ${ }^{20}$ Preliminary results of a study in 100 patients with AMN by Moser et al in the United States do not indicate an amelioration of clinical progression. ${ }^{33}$

Regrettably, the worldwide decision to conduct non-randomised studies has hampered the assessment of the efficacy of the dietary treatment tremendously. In retrospect, it is all too easy to argue that it was unethical to withhold from the patients a placebo controlled double blind study. However, there are several extenuating circumstances. When “Lorenzo's oil" was introduced it seemed unethical to withhold this apparently promising therapy from patients with the crippling and debilitating X-ALD. ${ }^{33}$ Other therapeutic options were lacking, and treatment with the GTO oil apparently induced a lowering of plasma VLCFAs and a statistically significant improvement in peroneal nerve conduction, suggesting that the oil might be efficacious to the central white matter as well. ${ }^{9}$ Furthermore, the prevalence of X-ALD was relatively low, and the phenotypic expression highly variable. ${ }^{57}$ As dangerous as it is to use historical controls, it would have been even riskier in the trials with "Lorenzo's oil", because the natural course in untreated patients was not exactly known. Until recently it was assumed that about 50\% of all patients with X-ALD would eventually develop cerebral variants, ${ }^{6}{ }^{9}$ but in a survey of 30 Dutch kindreds we found a lower risk of about $30 \%$, and predominance of the milder AMN phenotype (46\%). ${ }^{7}$ Nevertheless, we tried to compose a representative control group, but this was in vain: the patients who discontinued the dietary treatment were more severely affected, and those who rejected treatment were not keen on regular consultations or investigations.

The aggregate of the available data on the efficacy of "Lorenzo's oil" in adult patients with X-ALD and neurological symptoms suggests that the oil is not efficacious. Our results are in concordance with the findings of Aubourg et $a l^{11}$ and the preliminary results of the study by Moser et al. ${ }^{33}$ Even if treatment with "Lorenzo's oil" would be beneficial, several groups of investigators, including ours, were unable to detect these effects.

The accumulation of VLCFAs in brain lipids is associated with myelin instability and the inability of brain macrophages to remove the excess of VLCFAs. The accumulation of VLCFAs in lipids of the central white matter initiates a severe inflammatory reaction in the cerebral variants of $\mathrm{X}-\mathrm{ALD}$, resulting in demyelination. ${ }^{34}$ Therefore, it was reasoned that demyelination might be slowed down or halted if oleic and erucic acid reached the brain. Indeed, in 1989 Rizzo et al reported increased concentrations of erucic acid in the brain lipids of a patient with CCALD treated with "Lorenzo's oil" in comparison with an untreated patient, ${ }^{15}$ but Rasmussen et $a l^{35}$ and Poulos et $a l^{\beta 6}$ recently were unable to detect increased concentrations of erucic acid in the brain tissue of three patients with CCALD and two with $A M N$. This raises the question 
whether the dietary oils are able to pass the blood-brain barrier.

Patients with X-ALD who might still benefit from "Lorenzo's oil" are those without neurological deficits. Four of the six $(67 \%)$ neurologically asymptomatic patients with X-ALD we studied remained free of neuroinvolvement during the observation period (median follow up 2.1 years), and it seemed that the five "Addison only" patients on whom we unfortunately had limited data did not develop cerebral demyelination during a median follow up of 5.8 years. However, this observation period is too short to draw firm conclusions about the efficacy of "Lorenzo's oil" in these patients. Cappa et al reported that CNS demyelination occurred in only two of 11 asymptomatic patients during a follow up of 2 years. ${ }^{18}$ Asano et al found no neurological involvement after 2.5 years of dietary therapy in two asymptomatic patients, ${ }^{19}$ Aubourg et al were unable to detect neurological deterioration in five patients over an identical period, ${ }^{21}$ and Korenke et al observed no neuroinvolvement in seven patients over a 2 year period. ${ }^{20}$ Moser et al studied 53 neurologically asymptomatic patients with a mean age of 7.5 years at the start of treatment with "Lorenzo's oil". Preliminary analysis after a mean follow up of nearly 39 months showed that only $10 \%$ of the patients had signs of cerebral demyelination or had died, and that the others remained asymptomatic. ${ }^{37}$ These figures look promising, assuming that the risk for developing cerebral demyelination in X-ALD is high. However, the assessment of the efficacy of treatment with "Lorenzo's oil" in these patients is hampered as well by the absence of an untreated control group and our ignorance of the risk for developing each of the different phenotypes. At present, investigators in Europe (including members of our study group) and the United States are studying whether "Lorenzo's oil" can delay or prevent the onset of symptoms in a large group of neurologically asymptomatic patients with X-ALD. This study in supported by the BIOMED 2 programme of the European Commission and the United States Food and Drug Administration.

Data on the changes in endocrine function of the adrenal cortex and testis are sparse. In 1994 we reported that GTO and GTO/GTE therapy in all five patients with $\mathrm{AMN}$ improved neither adrenocortical nor gonadal function. ${ }^{38}$ In the present study we found no significant improvement in endocrine function of the adrenal cortex and testis, organs that probably are more easily accessible to oleic and erucic acid in the absence of a protective mechanism such as the blood-brain barrier. Many patients with X-ALD, ${ }^{27}{ }^{39}$ had extremely low concentrations of DHEA-S, the most abundantly produced adrenocortical hormone. This hormone seems to be involved in the modulation of the immune response, ${ }^{40}$ a response that is so excessive in the cerebral forms of X-ALD. ${ }^{34}$ The interaction between DHEA-S and the immune response in X-ALD deserves further research. For testis function, we recently found that most men with X-ALD (77\%) have clinical or subclinical testicular insufficiency, even in the absence of neurological deficits or adrenocortical insufficiency. ${ }^{27}$ The possible role of treatment with androgens in patients with X-ALD and testicular insufficiency merits further study.

Although treatment with "Lorenzo's oil" did not seem to be beneficial, we often found side effects such as mild increases in liver enzymes (55\%), thrombocytopenia (55\%), gastrointestinal complaints (14\%), and gingivitis $(14 \%)$. Furthermore, there was a decrease in $\mathrm{Hb}$ concentration and leucocyte count, and an increase in the plasma AP concentration. The reduction in platelets fortunately did not result in haemorrhagic diathesis. We recently reported that the thrombocytopenia may be due to platelet activation, resulting from an increase in the erucic acid concentration of lipids in the platelet membrane. ${ }^{41}$ In patients with counts below $80 \times 10^{9} / 1$ we lowered the GTE dose by 50 to $75 \%$, and again added GTE carefully until normal or near normal concentrations of C26:0 were achieved. Low platelet counts have often been found by others as well. $.^{19-21} 42-44$ Lymphocytopenia $^{45}$ and severely depressed natural killer cell activity ${ }^{46}$ were also reported. We do not know whether some of the side effects may have been due to low concentrations of essential fatty acids, or induced by reduction of the dietary fat intake; we are studying this at present.

The results of our study with "Lorenzo's oil" raise the question whether this therapy is efficacious at all, and whether a marginal therapeutic effect counterbalances the side effects. As other therapeutic options are virtually lacking for patients with X-ALD and impending neurological deficits, we still prescribe the GTO/GTE diet to neurologically asymptomatic patients with X-ALD, and to those with neurological symptoms who prefer to adhere to it. Bone marrow transplantation seems to be effective, but only in young boys with mild signs of cerebral demyelination. ${ }^{31} 32$ Other therapies such as immunosuppression, ${ }^{47}{ }^{48} \quad \beta$-interferon, ${ }^{49}$ and intravenous immunoglobulins ${ }^{50}$ were ineffective. The efficacy of "Lorenzo's oil" can only be assessed by a randomised, placebo controlled, double blind study. At present it seems very unlikely that such a trial will ever be conducted. Fortunately, Lu et al recently developed an X-ALD mouse model by gene targeting. ${ }^{51}$ The X-ALD mice exhibit biochemical defects equivalent to those found in patients with X-ALD, thus providing a manageable system for testing present and future therapeutic interventions.

Furthermore, two novel therapeutic approaches were described recently, and may be used in future trials. According to Singh et al, lovastatin, a 3-hydroxy-3-methylglutaryl coenzyme A reductase inhibitor used for the treatment of hypercholesterolaemia, also decreases plasma concentrations of VLCFAs in patients with X-ALD, and should be further evaluated. $^{52}$ Kemp et al reported that 4-phenylbutyrate, used against urea cycle disorders, lowers VLCFA concentrations by increasing $\beta$-oxidation in cultured cells from 
patients with X-ALD and the X-ALD knockout mice..$^{53}$

In conclusion, we were unable to detect a beneficial effect of treatment with "Lorenzo's oil" in patients with neurologically symptomatic X-ALD. On the contrary, the dietary therapy often caused adverse effects. We think that the oil should not be prescribed routinely to patients with X-ALD who already have neurological symptoms. However, it remains to be determined whether "Lorenzo's oil" can postpone neurological involvement in neurologically asymptomatic patients with X-ALD.

We thank $\mathrm{G} J$ Weverling for his advice on statistics and methodology and B W Ongerboer de Visser and M Vermeulen for their critical reading and helpful suggestions. We appreciate all the work done by A van Rooij, K van Ravensberg, L van Lint, and A Nijenhuis, Department of Pediatric Clinical Chemistry, Academic Medical Center, Amsterdam. We are very grateful to the referring physicians and members of the Dutch X-ALD/AMN Study Group, in particular: Academic Medical Center, Amsterdam: T Vulsma; University of Amsterdam, Amsterdam: J M Tager; University Hospital "Vrije Universiteit", Amsterdam: R B H Schutgens; University Hospital, Leiden: P'D MaaswinkelMooy; University Hospital, Nijmegen: A Keyser, L A H Monnens, B J Otten, G W Padberg, A G H Smals; University Hospital, Rotterdam: J B C de Klerk, M C B Loonen, H Stroink; St Elisabeth Ziekenhuis, Tilburg: L H Visser; University Hospital, Elisabeth Ziekenhuis, Tilburg: L H Visser; University Hospital, Utrecht: J H J Wokke. The study was financially supported by the "P
lands.

1 Singh I, Moser AE, Goldfischer S, et al. Lignoceric acid is oxidized in the peroxisome: implications for the Zellweger cerebro-hepato-renal syndrome and adrenoleukodystrophy. Proc Natl Acad Sci USA 1984;81:4203-7.

2 Wanders RJA, van Roermund CWT, van Wijland MJA, et al. $\mathrm{X}$ linked adrenoleukodystrophy: defective peroxisomal oxidation of very long chain fatty acids but not of very long chain fatty acyl-CoA esters. Clin Chim Acta 1987;165:3219 .

3 Lazo O, Contreras M, Hashmi M, et al. Peroxisomal ignoceroyl-CoA ligase deficiency in childhood adrenoleukodystrophy and adrenomyeloneuropathy. Proc Natl Acad Sci USA 1988;85:7647-51.

4 Powers JM. Adreno-leukodystrophy (adreno-testiculoleuko-myelo-neuropathic-complex). Clin Neuropathol 1985;4:181-99

5 Moser HW, Smith KD, Moser AB. X linked adrenoleukodystrophy. In: Scriver CR, Beaud A, Sly WS, et al, eds. The metabolic and molecular bases of inherited disease. 7 th ed. New York: McGraw-Hill, 1995:2325-49.

6 Aubourg P, Chaussain JL. Adrenoleukodystrophy presenting as Addison's disease in children and adults. Trends ing as Addison's disease in child

7 van Geel BM, Assies J, Weverling GJ, Barth PG. Predominance of the adrenomyeloneuropathy phenotype of X linked adrenoleukodystrophy in the Netherlands: survey of 30 kindreds. Neurology 1994;44:2343-6.

8 van Geel BM, Assies J, Wanders RJA, et al. X linked adrenoleukodystrophy: clinical presentation, diagnosis and therapy. I Neurol Neurosurg Psychiatry 1997;63:4-14.

9 Moser HW, Moser AB, Smith KD, et al. Adrenoleukodystrophy: phenotypic variability and implications for therapy. F Inher Metab Dis 1992;15:645-64.

10 Brown III FR, van Duyn MA, Moser AB, et al. Adrenoleukodystrophy: effects of of dietary restriction and of administration of carnitine and clofibrate on clinical status and plasma fatty acids. Fohns Hopkins Medical fournal 1982;151:164-72.

11 Tsuji S, Sano T, Ariga T, et al. Increased synthesis of hexacosanoic acid (C26:0) by cultured skin fibroblasts from patients with adrenoleukodystrophy (ALD) and adrenomyeloneuropathy (AMN). F Biochem 1981;90:1233-6.

12 Rizzo WB, Watkins PA, Phillips MW, et al. Adrenoleukodystrophy: oleic acid lowers fibroblast satuAdrenoleukodystrophy: oleic acid lowers fibroblast
rated C22-26 fatty acids. Neurology 1986;36:357-61.

13 Rizzo WB, Phillips MW, Dammann AL, et al. Adrenoleukodystrophy: dietary oleic acid lowers hexacosanoate levels. Ann Neurol 1987;21:232-9.

14 Moser AB, Borel J, Odone A, et al. A new dietary therapy for adrenoleukodystrophy: biochemical and preliminary clinical results in 36 patients. Ann Neurol 1987;21:240-9

15 Rizzo WB, Leshner RT, Odone A, et al. Dietary erucic acid therapy for X linked adrenoleukodystrophy. Neurology 1989;39:1415-22.

16 Odone A, Odone M. Lorenzo's oil: a new treatment for adrenoleukodystrophy. $\mathcal{F}$ Pediatr Neurosci 1989;5:55-61.

17 Uziel G, Bertini E, Bardelli P, et al. Experience on therapy of adrenoleukodystrophy and adrenomyeloneuropathy. Dev Neurosci 1991;13:274-9.

18 Cappa M, Cambiaso P, del Balzo P, et al. Adrenoleukodystrophy: clinical and therapeutic updating. In: Bhatt HR, James VHT, Besser GM, et al, eds. Advances in Thomas Addison's diseases. Vol 1. Bristol, UK: Journal of Endocrinology, 1994:171-80.
19 Asano J, Suzuki Y, Yajima S, et al. Effects of erucic acid therapy on Japanese patients with $\mathrm{X}$ linked adrenoleukodystrophy. Brain Dev 1994;16:454-8.

20 Korenke GC, Hunneman DH, Kohler W, et al. Glyceroltrioleate / glyceroltrierucate therapy in 16 patients with $\mathrm{X}$ chromosomal adrenoleukodystrophy/adrenomyeloneuropathy: effect on clinical, biochemical and neurophysiological parameters. Eur f Pediatr 1995;154:64-70.

21 Aubourg P, Adamsbaum C, Lavallard-Rousseau MC, et al. A 2 year trial of oleic and erucic acids (Lorenzo's oil) as treatment for adrenomyeloneuropathy. N Engl f Med 1993; 329:745-52.

22 Moser HW, Moser AB, Frayer KK, et al. Adrenoleukodystrophy: increased plasma content of saturated very long chain fatty acids. Neurology 1981;31:12416.

23 Moser HW, Moser AB, Kawamura N, et al. Adrenoleukodystrophy: elevated C26 fatty acid in cultured skin fibroblasts. Ann Neurol 1980;7:542-9.

24 Kurtzke JF. Rating neurologic impairment in multiple sclerosis: an expanded disability status scale (EDSS). Neurology 1983;33:1444-52.

25 Wade DT, Collin C. The Barthel ADL index: a standard measure of physical disability? International Disability Studies 1988;10:64-7.

26 van Swieten JC, Koudstaal PJ, Visser MC, et al. Interobserver agreement for the assessment of handicap in stroke patients. Stroke 1988;19:604-7.

27 Assies J, Gooren LJG, van Geel B, et al. Signs of testicular insufficiency in adrenoleukodystrophy and neurologically asymptomatic $\mathrm{X}$ linked adrenoleukodystrophy: a retrospective study. Int $f$ Andrology 1997;20:315-21.

28 van Geel BM, Koelman JHTM, Barth PG, et al. Peripheral nerve abnormalities in adrenomyeloneuropathy: a clinical and electrodiagnostic study. Neurology 1996;46:112-18.

29 Aramideh M, Hoogendijk JE, Aalfs CM, et al. Somatosensory evoked potentials, sensory nerve potentials and sensory nerve conduction in hereditary motor and sensory neuropathy type I. $\mathcal{f}$ Neurol 1992;239:277-83.

30 Aalfs CM, Koelman JHTM, Meyjes FE, et al. Posterior tibial and sural nerve somatosensory evoked potentials: a study in spastic paraparesis and spinal cord lesions. Electroencephalogr Clin Neurophysiol 1993;89:437-41.

31 Aubourg P, Blanche S, Jambaqué I, et al. Reversal of early neurologic and neuroradiologic manifestations of $\mathrm{X}$ linked adrenoleukodystrophy by bone marrow transplantation. $N$ Engl f Med 1990;322:1860-6.

32 Krivit W, Lockman LA, Watkins PA, et al. The future for treatment by bone marrow transplantation for adrenoleukodystrophy, metachromatic leukodystrophy, globoid cell leukodystrophy and Hurler syndrome. F Inherit Metab Dis 1995;18:398-412.

33 Moser HW, Borel J. Dietary management of X linked adrenoleukodystrophy. Annu Rev Nutr 1995;15:379-97.

34 Powers JM, Liu Y, Moser AB, et al. The inflammatory myelinopathy of adreno-leukodystrophy: cells, effector molecules, and pathogenetic implications. 7 Neuropathol Exp Neurol 1992;51:630-43.

35 Rasmussen M, Moser AB, Borel J, et al. Brain, liver, and adipose tissue erucic and very long chain fatty acid levels in adrenoleukodystrophy patients treated with glyceryl trierucate and trioleate oils (Lorenzo's oil). Neurochem Res 1994; 8:1073-82.

36 Poulos A, Gibson R, Sharp P, et al. Very long chain fatty acids in $\mathrm{X}$ linked adrenoleukodystrophy brain after treatment with Lorenzo's oil. Ann Neurol 1994;36:741-6.

37 Moser HW. Clinical and therapeutic aspects of adrenoleukodystrophy and adrenomyeloneuropathy. If Neuropathol Exp Neurol 1995;54:740-5.

38 Assies J, van Geel BM, Weverling GJ, et al. Endocrine evaluation during dietary therapy for adrenomyeloneuropathy. In: Bhatt HR, James VHT, Besser GM, et al, eds. Advances in Thomas Addison's diseases. Vol 1. Bristol, UK: Journal of Endocrinology 1994:191-8.

39 Korenke GC, Roth C, Krasemann E, et al. Variability of endocrinological dysfunction in 55 patients with $\mathrm{X}$ linked adrenoleukodystrophy: clinical, laboratory and genetic adrenoleukodystrophy: clinical, laboratory

40 Padgett DA, Loria RM. In vitro potentiation of lymphocyte activation by dehydroepiandrosterone, androstenediol and androstenetriol. F Immunol 1994;153:1544-52.

41 Konijnenberg A, van Geel BM, Sturk A, et al. Lorenzo's oil and platelet activation in adrenomyeloneuropathy and asymptomatic X linked adrenoleukodystrophy. Platelets 1998;9:41-8.

42 Unkrig CJ, Schröder R, Scharf RE. Lorenzo's oil and lymphocytopenia. N Engl f Med 1994;330:577.

43 Zierz S, Schröder R, Unkrig CJ. Thrombocytopenia induced by erucic acid therapy in patients with $\mathrm{X}$ linked adrenoleukodystrophy. Clin Invest 1993;71:802-5.

44 Zinkham WH, Kickler T, Borel J, et al. Lorenzo's oil and thrombocytopenia in patients with adrenoleukodystrophy. $N$ Engl F Med 1993;328:1126-7.

45 Toplak H, Wascher TC, Pleschko B, et al. Reduced stimulability of platelet surface adhesion molecules under treatment with Lorenzo's oil. F Inherit Metab Dis 1994;17: $628-9$.

46 Sedlmayr P, Pleschko B, Paschke E, et al. Severely depressed natural killer cell activity of patients with adrenoleukodystrophy under treatment with Lorenzo's oil. F Inherit Metab Dis 1995;18:101-2. 
47 Stumpf DA, Hayward A, Haas R, et al. Adrenoleukodystrophy: failure of immunosuppression to prevent neurological progression. Arch Neurol 1981;38:48-

48 Naidu S, Bresnan MJ, Griffin D, et al. Childhood adrenoleukodystrophy: failure of intensive immunosuppression to arrest neurological progression. Arch Neurol 1988;45:846-8.

49 Korenke GC, Christen H-J, Kruse B, et al. Progression of X linked adrenoleukodystrophy under interferon- $\beta$ therapy. $\mathcal{F}$ Inherit Metab Dis 1997;20:59-66.
50 Cappa M, Bertini E, del Balzo P, et al. High dose immunoglobulin IV treatment in adrenoleukodystrophy. $\mathcal{F}$ (N) 69-70.

$51 \mathrm{Lu} \mathrm{J-F}$, Lawler AM, Watkins PA, et al. A mouse model for X linked adrenoleukodystrophy. Proc Natl Acad Sci USA 1997;94:9366-71.

52 Singh I, Khan M, Key L, et al. Lovastatin for X linked adrenoleukodystrophy. $\mathrm{N} \mathrm{Engl} \mathcal{F}$ Med 1998;339:702-3.

$53 \mathrm{Kemp} \mathrm{S}$, Wei H-M, Lu J-F, et al. Gene redundancy and pharmacological gene therapy: implications for $\mathrm{X}$ linked adrenoleukodystrophy. Nat Med 1998;4:1261-8.

\section{NEUROLOGICAL STAMP}

\section{Ivan Petrovich Pavlov (1849-1936)}

Although trained as a physician Pavlov always worked as an experimental laboratory scientist. He only had contact with patients at the end of his career. Under the influence of the well known clinician Sergei Petrovich Botkin he developed a commitment to nervous, as opposed to humoral explanations for bodily functions. By 1883 he had developed his theory of "nervism" which he defined as a physiological theory which tries to prove that the nervous system controls the greatest possible number of bodily functions. With the development of aseptic methods of surgery at that time, Pavlov was able to develop surgical methods for physiological research. One of his teachers, Heidenham, had previously made a stomach pouch from which he could obtain pure gastric juice free from food but in the formation of the pouch Heidenham had destroyed the nerve supply to the pouch. Pavlov had a great skill as an experimental surgeon. He developed a small part of the stomach, the "Pavlov pouch" as well as chronic external salivary, biliary, and pancreatic fistulae for his fundamental study of gastric physiology, publication of which won him the Nobel Prize in 1904. Pavlov demonstrated that secretory nerve fibres of the pancreas and stomach were in the vagus nerve.

With his later work on conditioned reflexes (1912), the effect sensory or psychic stimuli have on reflex actions, Pavlov showed that stimuli such as a musical note, a bright colour, a strong odour, skin stimuli, or an electric shock if previously associated with the sight of food, caused salivation. At the sound of a given note this would cease if the note was raised or lowered even by a quarter tone. Pavlov thought that the conditional or acquired reflexes were associated with different areas of the cerebral cortex. This led to a new psychology oriented school of physiology and stimulated ideas of many aspects of human behaviour being the result of conditioning. In his acceptance of the Nobel Prize for his work on digestive physiology in 1904, Pavlov chose to speak on his work on conditioned reflexes.

Pavlov further extended this sphere of his research interest into human psychophysiology and psychopathology. In the late 1920s he began making observations on patients in a psychiatric hospital and attempted to explain disorders that he saw in terms of his work on conditioning.

Pavlov criticised communism and in 1922 requested and was refused permission to move his laboratory abroad. After expulsion of priests' sons from the Medical Academy,

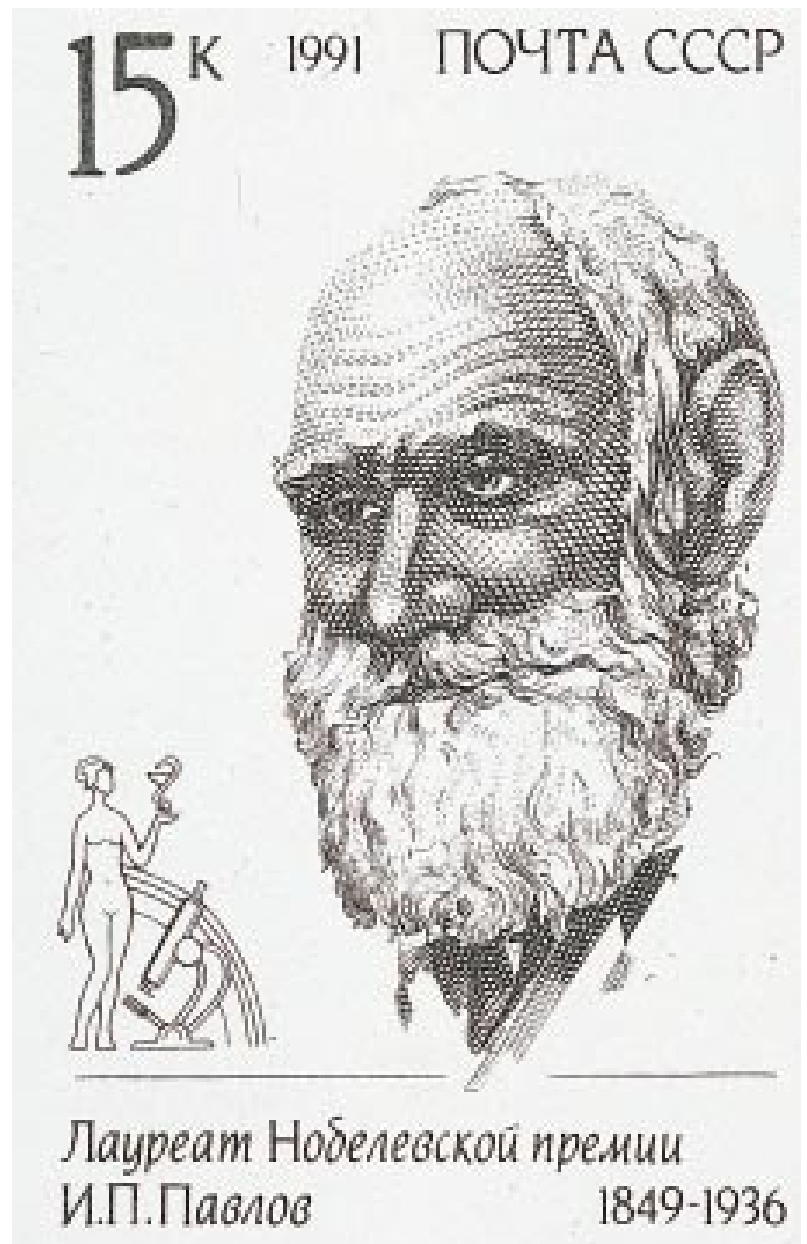

Pavlov, who was also the son of a poor village priest and himself a former seminary student resigned in protest from the Chair of Physiology but his research continued to be supported by State funds.

Pavlov has been philatelically honoured on several occasions. He is shown here on a Russian stamp of 1991 (Stanley Gibbons 6253, Scott 5999) in a series commemorating Russian winners of the Nobel Prize.

L F HAAS 